\title{
Evidence of woven bone formation in carotid artery plaques
}

\section{Mirzaie Masoud ${ }^{1 *}$, Zaur Guliyev ${ }^{1}$, Michael Schultz², Peter Schwartz ${ }^{2}$, Johann Philipp Addicks ${ }^{3}$ and Sheila Fatehpur ${ }^{1}$}

\author{
1'Department of Vascular Surgery, Lippe-Lemgo Hospital, University clinics OWL, Germany \\ ${ }^{2}$ Institute for Anatomy, University of Göttingen, Göttingen, Germany \\ ${ }^{3}$ Institute of Neuroradiology, Lippe-Lemgo Hospital, University clinics OWL, Germany
}

\section{Abstract}

Objective: Plaque morphology plays an important prognostic role in the occurrence of cerebrovascular events. Echolucent and heterogeneous plaques, in particular, carry an increased risk of subsequent stroke. Depending on the quality of the plaque echogenicity based on B-mode ultrasound examination, carotid plaques divide into a soft lipid-rich plaque and a hard plaque with calcification. The aim of this study was to investigate structural changes in the basement membrane of different carotid artery plaque types.

Patients and methods: Biopsies were taken from 10 male patients (average age; $75+1$ years) and 7 females $(68+3$ years). The study population included patients suffering from a filiform stenosis of the carotid artery, 8 patients with acute cerebrovascular events and 9 with asymptomatic stenosis. Scanning electron and polarised light microscopic investigations were carried out on explanted plaques to determine the morphology of calcified areas in vascular lesions.

Results: By means of scanning electron microscopy, multiple foci of local calcification were identified. The endothelial layer was partially desquamated from the basement membrane and showed island-like formations. Polarised light microscopy allows us to distinguish between soft plaques with transparent structure and hard plaques with woven bone formation.

Conclusion: The major finding of our study is the presence of woven bone tissue in hard plaques of carotid arteries, which may result from pathological strains or mechanical overloading of the collagen fibers. These data suggest a certain parallel with sclerosis of human aortic valves due to their similar morphological characteristics.

\section{More Information}

*Address for Correspondence: Masoud Mirzaie, Professor, MD, Head of the Department of Vascular Surgery, University Hospital of Lemgo, Rintelner Straße 85, 32657 Lemgo, Rintelner Str. 8532657 Lemgo, Germany, Tel: +49-5261/26-4142; Fax: +49-5261/26-4669; Email: masoud.mirzaie@klinikum-lippe.de

Submitted: November 24, 2020

Approved: January 04, 2021

Published: January 05, 2021

How to cite this article: Masoud M, Guliyev Z, Schultz M, Schwartz P, Addicks JP, et al. Evidence of woven bone formation in carotid artery plaques. J Cardiol Cardiovasc Med. 2021; 6: 001-006.

\section{DOI: 10.29328/journal.jccm.1001108}

Copyright: @ 2021 Masoud M, et al. This is an open access article distributed under the Creative Commons Attribution License, which permits unrestricted use, distribution, and reproduction in any medium, provided the original work is properly cited.

Keywords: Woven bone; Carotis artery plaques

Check for updates

OPEN ACCESS

\section{Introduction}

In addition to the degree of stenosis, analysis of the internal morphological structure of the plaques is becoming an increasingly important, contributory factor in stroke risk. Compared with calcium-containing "hard plaques", plaques with a high lipid content are associated with an increased risk of ipsilateral cerebral ischemia [1,2]. Most of the trials emphasizing the relationship between plaque echo structure and stroke risk are based on high-resolution ultrasound (US) using a visual method of classification $[3,4]$. To date, the plaques of carotid arteries are divided into friable, fibroatheromatous and atheromatous forms [5]. In a previous study on explanted human aortic valves, calcified areas showed characteristic morphological features of woven bone formation [6]. In this report, we describe the morphology of plaques of carotid arteries using polarised light microscopy in addition to conventional electron microscopical techniques. Especially, we were interested in the identification of bony structures mimicking valve calcification.

\section{Materials and methods}

\section{Tissue biopsies}

Tissue samples from the carotid artery were taken from patients undergoing routine endarteriectomy of the carotid artery in operations at the Department of Thoracic, Heart and Vascular Surgery. 17 biopsies were taken from 10 male patients (average age; $75+1$ years) and 7 females $(68+3$ years). The study population included patients suffering from filiform stenosis of the carotid artery. In 9 cases, stenosis of the carotid artery occurred without any symptoms, in 8 patients 
signs of circulatory disturbance of the vertebrobasila system or the carotid artery were observed. Table 1 summarizes the basic data of the study patients.

\begin{tabular}{|c|c|c|}
\hline Male $(n=10$, age: $75+1$ years $)$ & \multicolumn{2}{|c|}{ Female $(n=7$, age: $68+3$ years $)$} \\
\hline \multicolumn{3}{|l|}{ Symptoms } \\
\hline & vertigo & $4(23, .5 \%)$ \\
\hline & Acoustic Aphasia & $3 / 17.6 \%)$ \\
\hline & Motoric Aphasia & $1(5.8 \%)$ \\
\hline & Paresis & \\
\hline Previous surgeries & TEA of carotid artery & $1(5.8 \%)$ \\
\hline \multicolumn{3}{|l|}{ Concomitant Diseases } \\
\hline & $\mathrm{CHD}$ & $3(17.6 \%)$ \\
\hline & HLP & $5(29.4 \%)$ \\
\hline & Smoking & $9(52.9 \%)$ \\
\hline & Arteial Hypertension & $10(58.8 \%)$ \\
\hline Statins & & $7(41.1 \%)$ \\
\hline
\end{tabular}

Continous variables are presented as mean + standard deviation. Categorical variables are presented as an absolute percentage.

Abbreviations: HLP: Hyperlipoproteinaemia, CHD: Coronary Heart Disease, TEA: Thrombendartriektomy

\section{CT-Angiography}

All patients underwent preoperative CT angiography in a 64-row CT scan. For this purpose, the bolus-triggered examination technique was used with a bolus of $80-100 \mathrm{ml}$ $\mathrm{KM}$ at flow rates of 3-5 ml/s with scan velocities up to almost $10 \mathrm{~cm} / \mathrm{s}$ after reaching an enhancement of $100 \mathrm{HU}$ with a delay of 6-12 s. The source recordings were then reconstructed in 3-D technique.

\section{Scanning electron microscopy}

In order to reveal the surface morphology of the explanted valves, scanning electron microscopy was performed. Specimens from valve leaflets were fixed for $6 \mathrm{~h}$ in a solution containing $2.5 \%$ glutaraldehyde and $0.2 \mathrm{M}$ cacodylate. Afterwards, samples were dehydrated in a series of increasing concentrations of alcohol. After critical point drying, all samples were sputtered with gold-palladium. Samples were visualized using the digital scanning microscope Zeiss DSM 960 .

\section{Polarised light microscopy}

To evaluate the presence of woven bone tissue in calcified areas of pathologically altered aortic and mitral valves, polarized light microscopy was performed. To prepare thin ground sections from non-decalcified materials for polarised light microscopy, a special technique was established based upon the method of plastination developed by Hagens, et al. and modified for histological purposes by Schultz and Drommer [7,8]. Samples were dehydrated using ascending concentration steps of alcohol, washed in methyl chloride as an intermediate solution for the exchange of substances and embedded in epoxy resin Biodur ${ }^{\circledR}$. Unstained thin ground sections (30, 50 and $70 \mu \mathrm{m})$ were prepared and viewed in (the) transmitted plane and in polarised light using a hilfsobject red $1^{\text {st }}$ order (quartz), equipped with photo documentation $[9,10]$.

\section{Ethics}

The Ethics Committee of the University of Göttingen has approved the investigations on expanted carotid artery plaques on patients to determine of woven bone formation in carotid artery (reference: 25/6/2010).

\section{Results}

\section{CT-Angiography findings}

The CT-angiography of a patient with soft plaque shows bilateral high-grade internal carotid stenosis due to artheromatous plaque. Compared to the vascular environment, hypodense concentric plaque is shown intraluminally. The lumen itself contrasts hyperdensely calcified plaque is not detectable (Figure 1a).

The CT angiography of a patient with hard plaque also shows high-grade internal carotid stenosis due to calcifying plaques. There is hardly any evidence of hypodense internal plaques. A small hyperdensely contrasted lumen with extensive, bizarre, fissured calcifications, sometimes with adjacent hardening artefacts, is shown (Figure 1b).

\section{Scanning electron microscopical findings}

Figure 2a demonstrates a conserved endothelium, whereas the surface of the observed plaques was uneven and partially broken open. Endothelial cells appeared to be hypertrophied (Figure 2a). The endothelial layer was divided by small islands. At higher magnification, multiple initial tearings were observed and the matrix was uncovered. In these areas,

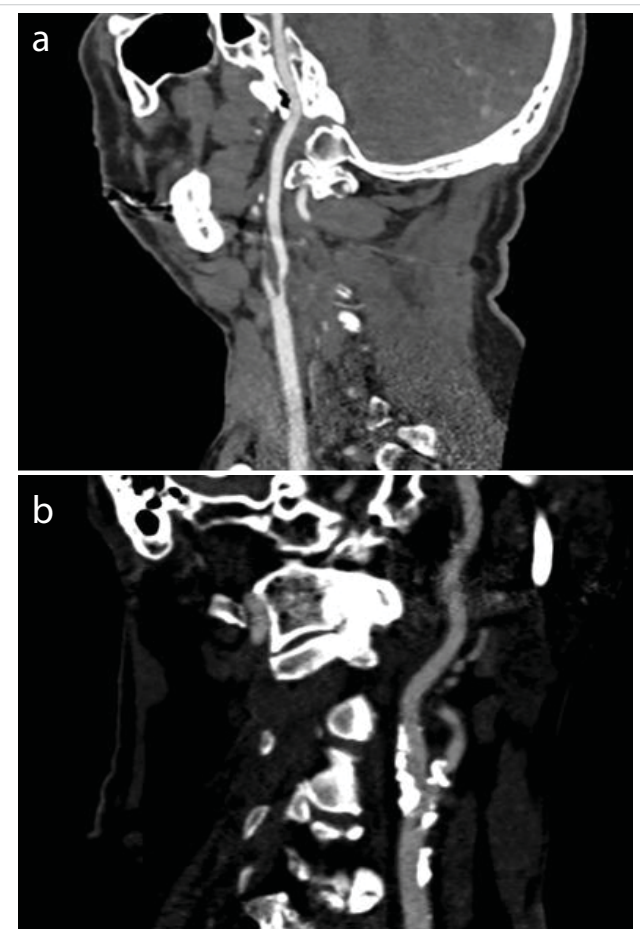

Figure 1: a: CT-Angiographie finding from a patient with soft plaque: intraluminally hypodensic concentric plaque without calcified areas. b: CT-Angiographie finding from a patient with hard plaque: high-grade internal carotid stenosis with extensive, bizarrely fissured calcifications. 
incorporation of erythrocytes into the extracellular matrix was observed. Some large plaques were covered with a thin layer of endothelial cells (Figure 2b,c).

\section{Polarised light microscopical findings}

Recent techniques employing polarised light microscopy as described by Bloebaum, et al. are potentially useful to evaluate the presence of woven bone tissue [10]. Tissue samples from normal artery walls revealed no pathological alterations.

Polarised light microscopy enabled) a distinction between two different types of plaques. In transmitted plane light,

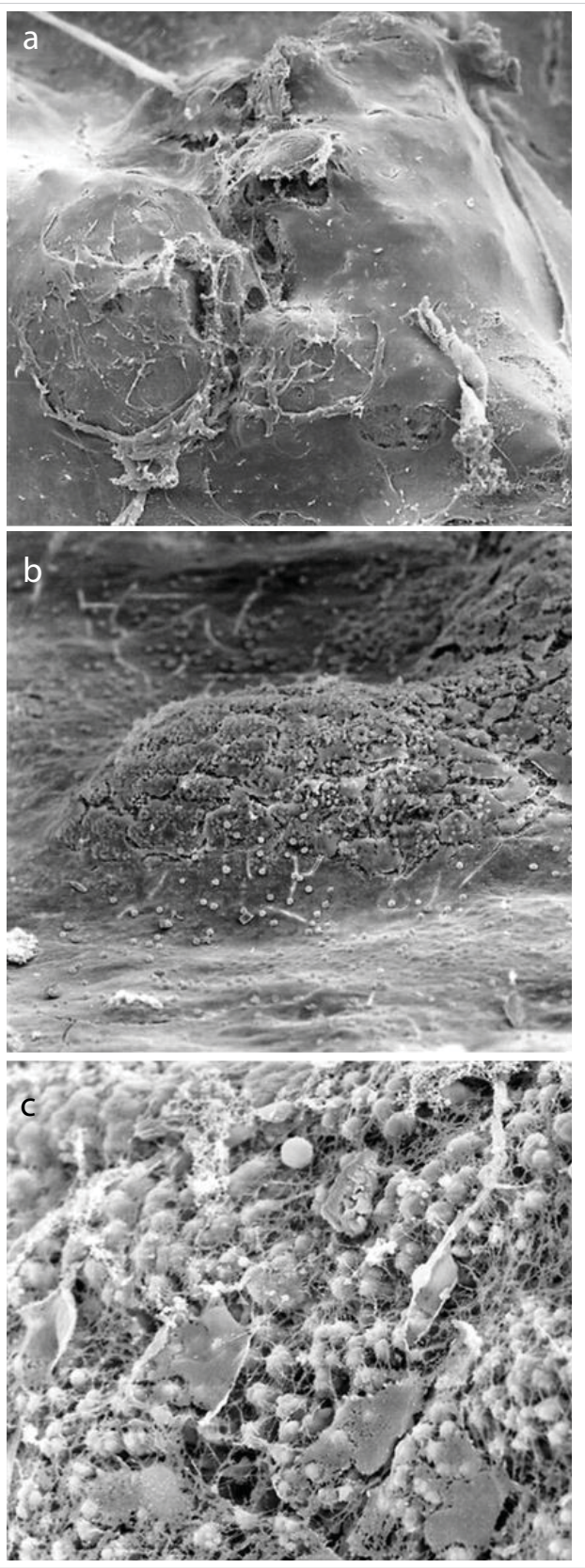

Figure 2: a: Scanning electron microscopical findings of internal carotid plaque ( $50 \mathrm{x}$ magnification): the surface of the observed plaques was uneven and partially broken. b: Scanning electron microscopical findings of internal carotid plaque (200 x magnification): multiple initial tearings and the uncovered extracellular matrix. c: Scanning electron microscopical findings of internal carotid plaque (1000 $\mathrm{x}$ magnification): incorporation of erythrocytes into the extracellular matrix with partially thin layer of endothelial cells. the collagenous fibers as well as intravascular deposits were transparent. Using a hilfsobject red $1^{\text {st }}$ order, deposits looked lucent (Figure 3a,b). When unstained thin ground sections of pathologically altered samples of the second group were viewed in transmitted plane light, the intravalvular localized inhomogeneous inclusions appeared as a secondary substance of yellowish or blue color (Figure 3c). In regular light, the character of this substance could be described as fibrous resembling natural woven bone. At high magnification (620 x), bundles of collagen fibers were detected (Figure $3 d$ ).

We detemine paque group 1 with evidence of woven bone formation in 4 patients with symptoms and 4 asymptomatic patients. The palque group without evidence of woven bone formations was found predominantly in symptomatic patients (5 symptomatic versus 3 asymptomatic patients). Nevertheless, a clinical correlation between plaque form and symptomatology cannot be deduced.

\section{Discussion}

Thromboembolism from extra-cranial atherosclerosis accounts for the neurological deficit in approximately half of the patients with ischaemic stroke [9]. Selection for eradicating interventions is conventionally determined by measuring luminal stenosis that results from in situ atheromatous plaques [7-9]. Apart from the degree of stenosis, plaque morphology has emerged in recent years as an important contributory factor in stroke risk [3]. During onset of thrombembolic events in arteresclerosis, vulnerable plaques play an important role [10]. Histological studies of coronary atherosclerosis suggest that plaques can be identified by thin fibrous caps that overlie large, often necrotic lipid cores $[11,12]$. A multimodal assessment of plaque vulnerability involving the combination of systemic markers, new imaging methods, for example, ultrasonic investigation, ct-imaging and MRI that target inflammatory and thrombotic components, and the potential of emerging therapies may lead to a new stratification system for atherothrombotic risk and to a better prevention of atherothrombotic stroke [3-5,13-16]. Mechanisms of plaque rupture have been extensively studied and several parameters have been found to interact: extracellular matrix, inflammatory cells, gelatinases, stromelysins, matrilysin and MMp expression induced by oxidised lipids etc. [17-22].

In a previous study on degenerative explanted human aortic valves, calcified areas were detected with characteristic morphological features of woven bone formation. Pathologically altered heart valves appear to exhibit distinct stages of desmal osteogenesis [6]. Formation of woven bone formation indicates an inappropriate biomechanical stress of collagenous fibers, potentially due to malsynthesis.

In this paper we report on our systematic SEM and polarised light microscopical investigations on explanted human carotid plaques. In all the samples, we detected uniform changes in the endothelium and the basement membrane. The endothelial 

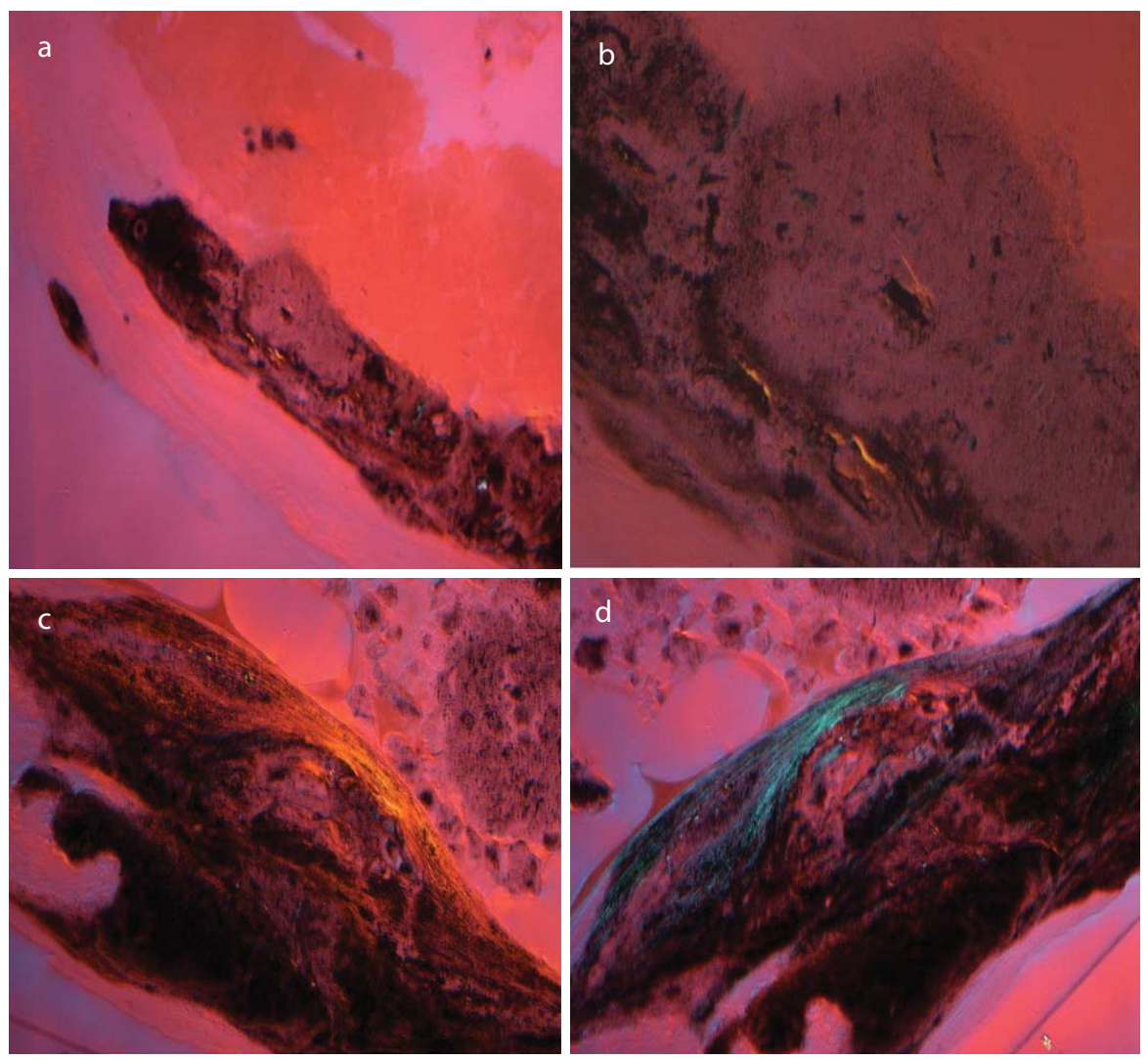

Figure 3: a: Polarised light microscopical findings of internal carotid plaque: impressed hyaline, collagenous fibers and intravascular deposites apear in transmitted plane light transparent. b: Polarised light microscopical findings of internal carotid plaque: lucent deposits using a hilfsobject red 1st order. c: Polarised light microscopical findings of internal carotid plaque: the intravalvular localized inhomogeneous inclusions of calcified plaques represented as a secondary substance of yellowish or blue color. $\mathbf{d}$ : Polarised light microscopical findings of internal carotid plaque: at high magnification (620 $\mathrm{x}$ ) bundles of collagen fibers and blue color structures resembling natural woven bone.

cells often showed hyperplasia with loose binding to each other. Rarely, an endothelial layer was complete. The loss of endothelial cells may expose the extracellular matrix, which obviously sets various pathological processes in motion [2326]. The increased activation of matrix metalloproteinases in pathologically altered human cardiac valves emphasizes the crucial role of the extracellular matrix in the development of this disease [26]. Similar mechanisms are held responsible for rupture of atherosclerotic plaques [21].

Polarised light microscopy identified two different types of plaques, a soft one with a transparent structure and without the presence of bone tissue, called soft plaques, and hard plaques with woven bone structures. These findings are probably identical with echolucent (predominant lipid core) und echo-opaque plaques (predominantly fibrous tissue/ calcium) as identified by sonography and magnetic resonance tomography [27-31]. The major finding of our study is the presence of woven bone tissue in hard carotid plaques. Dystrophic calcification first described by Mönckeberg is the most common pathological finding in surgically explanted valves [32]. Virchow recognized already that the mineralization of the walls of arteries in atherosclerosis is a process of ossification and not only a process of calcification [33]. Several case reports and clinical studies have identified bone proteins in ossified areas. The studies on the pathophysiology of heterotopic enchondral ossification in atherosclerotic plaque of arterial walls showed that osteoprogenitor cells resemble microvascular pericytes [34,35]. Myofibroblast-like cells, situated throughout the fibroid layer of cardiac valves and cultured in vitro, are capable of phenotypic differentiation into osteoblast-like cells [36,37]. Many authors, therefore, suggest the existence of a population of ossifying cells in both aorta and cardiac valves [34-38]. Atherosclerosis is a multifactorial, multistep disease that involves chronic inflammation as well as oxidation [34]. Detection of woven bone structure in pathologically altered human aortic valves and in carotid plaques indicates an additional pathogenic factor in the onset of atherosclerosis. In accordance with findings on human aortic valves, malsynthesis of collagenous fibers might contribute to pathogenesis of atherosclerosis of carotid arteries. Dystrophic calcification is a passive process in degenerating connective tissue, whereas heterotrophic ossification is an active process of abnormal tisssue repair. During the process of desmal ossification, collagen is produced which can easily be diagnosed by light microscopic examination using polarised light. The newly formed primitive woven bone separates intravalvular inclusions from surrounding collagen fibers [6].

The detection of woven bony tissue suggests that inadequate strain favours the mineralization of carotid plaques. The altered mechanical environment and the 
associated abnormal hemodynamic flow conditions may induce proliferative stimuli for the endothelium resulting in a combination of degenerative and hyperplasic responses. Pathological strains on the aortic walls finally result in the development of primary bundle bone-like tissue. The findings of the present and prior studies document the complex nature of atherosclerosis. Further studies employing biochemical and biophysical techniques may reveal the pathological basis of the underlying bony development and will help to understand the contribution of calcification in the context of atherosclerosis.

\section{Conclusion}

The submitted study demonstrated 2 fundamentally different carotid plaques. Whereas the first group shows a distinct of woven bone tissue, the second group completely lacks this tissue. In the past, we could demonstrate the woven bone tissue in degenerated aortic and mitral valves. we assume that, in addition to flow physiocomechanical changes in blood flow, collagen metabolism disorders play a central role in the pathogenesis of carotid stenosis.

\section{References}

1. Moroni F, Ammirati E, Norata GD, Magnoni M, Camici PG. The role of monocytes and macrophages in human atherosclerosis, plaque neoangiogenesis, and atherothrombosis. Mediators Inflamm. 2019; 7434376. PubMed: https://pubmed.ncbi.nlm.nih.gov/31089324/

2. Zhu G, Hom J, Li Y, Jiang B, Rodriguez F, et al. Carotid plaque imaging and the risk of atherosclerotic cardiovascular disease. Cardiovasc Diagn Ther. 2020; 10: 1048-1067.

PubMed: https://pubmed.ncbi.nlm.nih.gov/32968660/

3. Johri AM, Nambi V, Naqvi TZ, Feinstein SB, Kim ESH, et al Recommendations for the assessment of carotid arterial plaque by ultrasound for the characterization of atherosclerosis and evaluation of cardiovascular risk. JASE. 2020; 33: 917-933.

PubMed: https://pubmed.ncbi.nlm.nih.gov/32600741/

4. Momjian S, Lalive P, Sztajzel R. Carotid plaque: comparison between visual and grey-scale median analysis. Ultrasound Med Biol. 2003; 29: 961-966.

PubMed: https://pubmed.ncbi.nlm.nih.gov/12878241/

5. Mughal MM, Khan MK, DeMarco JK. Symptomatic and asymptomatic carotid artery plaque, Expert Rev Cardiovasc Ther. 2011; 9: 1315-1330. PubMed: https://www.ncbi.nlm.nih.gov/pmc/articles/PMC3243497/

6. Mirzaie M, Schultz M, Schwartz P. Evidence of woven bone formation in heart valve disease. Ann Thorac Cardiovasc Surg. 2003; 9: 163-169. PubMed: https://pubmed.ncbi.nlm.nih.gov/12875637/

7. Von Hagens G. Impregnation of soft biological specimens with thermostetting resins and elastomers. Anat Rec. 1979; 94: 247-255. PubMed: https://pubmed.ncbi.nlm.nih.gov/464325/

8. Schultz M, Drommer R. Possibilities of preparation production from the craniofacial area for macroscopic and microscopic examination using new plastics techniques. Exp Mund-KieferGesichts Chir. 1983; 28: 95-97.

9. Meershoek A, van Dijk RA, Verhage S. Hamming JF, van den Bogaerdt AJ, et al. Histological evaluation disqualifies IMT and calcification scores as surrogates for grading coronary and aortic atherosclerosis. Int J Cardiol. 2016; 224: 328-334.

PubMed: https://pubmed.ncbi.nlm.nih.gov/27668706/

10. Nambi V, Chambless L, Folsom AR. He M, Hu Y, et al. Carotid intima-media thickness and presence or absence of plaque improves prediction of coronary heart disease risk: The ARIC (Atherosclerosis risk in communities) study. J Am Coll Cardiol. 2010; 55: 1600-1607. PubMed: https://pubmed.ncbi.nlm.nih.gov/20378078/

11. Toutouzas K, Karanasos A, Tousoulis D. Optical coherence tomography for the detection of the vulnerable plaque. Eur Cardiol. 2016; 11: 90-95.

PubMed: https://pubmed.ncbi.nlm.nih.gov/30310454/

12. Hua Y. Chinese guidance on vascular ultrasound examination in stroke. Chin J Med Ultrasound. 2015; 8: 599-610.

13. Pelisek J, Wendorff $\mathrm{H}$, Wendorff $\mathrm{C}$. Age-associated changes in human carotid atherosclerotic plaques. Ann Med. 2016; 48: 541-551.

PubMed: https://pubmed.ncbi.nlm.nih.gov/27595161/

14. Jinnouchi $H$, Sato $Y$, Sakamoto $A$, Cornelissen $A$, Mori $M$, et al Calcium deposition within coronary atherosclerotic lesion: Implications for plaque stability. Finn Atherosclerosis. 2020; 306: 85-95.

PubMed: https://pubmed.ncbi.nlm.nih.gov/32654790/

15. Antipova D, Eadie L, Makin S. The use of transcranial ultrasound and clinical assessment to diagnose ischaemic stroke due to large vessel occlusion in remote and rural areas, PLoS One. 2020; 15: e0239653. PubMed: https://pubmed.ncbi.nlm.nih.gov/33007053/

16. Ahluwalia N, Genoux A, Ferrieres J, Perret B, Carayol M, et al. Iron status is associated with carotid atherosclerotic plaques in middleaged adults. J Nutr. 2010; 140: 812-816.

PubMed: https://pubmed.ncbi.nlm.nih.gov/20181783/

17. Sun B, Zhao H, Liu X, Lu Q, Zhao X, et al. Elevated hemoglobin A1c is associated with carotid plaque vulnerability: Novel findings from magnetic resonance imaging study in hypertensive stroke patients. Sci Rep. 2016; 6: 33246.

PubMed: https://pubmed.ncbi.nlm.nih.gov/27629481/

18. Brophy ML, Dong Y, Wu H, Rahman HNA, Song K, et al. Eating the dead to keep atherosclerosis at bay. Front Cardiovasc Med. 2017; 4: 2. PubMed: https://pubmed.ncbi.nlm.nih.gov/28194400/

19. Vaidya K, Martínez G, Patel S. The role of colchicine in acute coronary syndromes. Clin Ther. 2019; 41: 11-20.

PubMed: https://pubmed.ncbi.nlm.nih.gov/30185392/

20. Ruddy JM, Ikonomidis JS, Jones JA. Multidimensional contribution of matrix metalloproteinases to atherosclerotic plaque vulnerability: Multiple mechanisms of inhibition to promote stability. J Vasc Res. 2016; 53: 1-16.

PubMed: https://pubmed.ncbi.nlm.nih.gov/27327039/

21. Alhendi AMN, Patrikakis M, Daub CO, Kawaji H, Itoh M, et al. Promoter usage and dynamics in vascular smooth muscle cells Exposed to fibroblast growth factor-2 or interleukin-1ß. Sci Rep. 2018; 8: 13164. PubMed: https://pubmed.ncbi.nlm.nih.gov/30177712/

22. Veseli BE, Perrotta P, De Meyer GRA, Roth L, der Donckt CV, et al Animal models of atherosclerosis. Eur J Pharmacol. 2017; 816: 3-13. PubMed: https://pubmed.ncbi.nlm.nih.gov/28483459/

23. Nikkari ST, O’Brien KD, Ferguson M, Hatsukami T, Welgus HG, et al. Interstitial collagenase (MMP-1) expression in human carotid atherosclerosis. Circulation. 1995; 92: 1393-1398.

PubMed: https://pubmed.ncbi.nlm.nih.gov/7664418/

24. Chen X, Cao X, Jiang H, Che X, Xu X, et al. SIKVAV-modified chitosan hydrogel as a skin substitutes for wound closure in mice. Molecules. 2018; 23: 2611.

PubMed: https://pubmed.ncbi.nlm.nih.gov/30314388/

25. Cheng S, Wang D, Jin Ke J, Ma L, Zhou J, et al. Improved in vitro angiogenic behavior of human umbilical vein endothelial cells with oxidized polydopamine coating. Colloid Surface B. 2020; 194: 111176. PubMed: https://pubmed.ncbi.nlm.nih.gov/32540767/

26. Chia JS, Du JL, Hsu WB, Sun A, Chiang CP, et al. Inhibition of metastasis, angiogenesis, and tumor growth by Chinese herbal cocktail Tien-Hsien Liquid. BMC Cancer. 2010; 10: 175.

PubMed: https://pubmed.ncbi.nlm.nih.gov/20429953/ 
27. Latha S, Samiappan D, Kumar R. Carotid artery ultrasound image analysis: A review of the literature. Proc Inst Mech Eng H. 2020; 234: 417-443.

PubMed: https://pubmed.ncbi.nlm.nih.gov/31960771/

28. Saxena A, Kwee Ng EY, Teik S. Imaging modalities to diagnose carotid artery stenosis: progress and prospect, Bio Med Eng. 2019; 18: 66. PubMed: https://pubmed.ncbi.nlm.nih.gov/31138235/

29. Banaei $A$. The comparison between digital subtraction angiography, CT angiography, and doppler ultrasonography in evaluation and assessment of carotid artery stenosis. Ann Mil Health Sci Res. 2017; 15: e61661.

30. Correia M, Maresca D, Goudot G, Villemain O, Bizé A, et al Quantitative imaging of coronary flows using 3D ultrafast doppler coronary angiography. Phys Med Biol. 2020; 65: 105013.

PubMed: https://pubmed.ncbi.nlm.nih.gov/32340010/

31. Morbiducci U, Kok AM, Kwak BR. Atherosclerosis at arteria bifurcations: Evidence for the role of haemodynamics and geometry. Thrombosis and Haemostasis. 2016; 115: 484492.

PubMed: https://pubmed.ncbi.nlm.nih.gov/26740210/

32. Monckeberg JG. Normal histological construction and sclerosis of aortic valves. Virchows Archiv Pathol Anat Physiol. 1904; 176: 472-514.
33. Virchow R. Cellular pathology: as based upon physiological and pathological histology. Nutr Rev. 1989; 47: 23-25.

PubMed: https://pubmed.ncbi.nlm.nih.gov/2649802/

34. Yang $\mathrm{P}$, Troncone L, Augur ZM, Kim SSJ, McNeil ME, et al. The role of bone morphogenetic protein signaling in vascular calcification. Bone. 2020; 115542

PubMed: https://pubmed.ncbi.nlm.nih.gov/32736145/

35. Sun $\mathrm{M}$, Chang $\mathrm{Q}$, Xin $\mathrm{M}$. Endogenous bone morphogenetic protein 2 plays a role in vascular smooth muscle cell calcification induced by interleukin 6 in vitro. Int J Immunopathol Pharmacol. 2017; 30: 227-237. PubMed: https://pubmed.ncbi.nlm.nih.gov/28134597/

36. Nagy E, Eriksson P, Yousry M, Caidahl K, Ingelsson E, et al. Valvular osteoclasts in calcification and aortic valve stenosis severity. Int $\mathrm{J}$ Cardiol. 2013; 168: 2264-2271.

PubMed: https://pubmed.ncbi.nlm.nih.gov/23452891/

37. Low EL, Baker AH, Bradshaw AC. TGF $\beta$, smooth muscle cells and coronary artery disease: a review. Cell Signal. 2019; 53: 90-101. PubMed: https://pubmed.ncbi.nlm.nih.gov/30227237/

38. Shioi A, Ikari Y. Plaque calcification during atherosclerosis progression and regression. J Atheroscler Thromb. 2018; 25: 294-303.

PubMed: https://pubmed.ncbi.nlm.nih.gov/29238011/ 